\title{
Optimal Scheduling of Reliability Development Activities
}

\author{
John Quigley and Lesley Walls \\ Dept Management Science, University of Strathclyde \\ Glasgow, Scotland \\ Walter Johnston \\ BAE SYSTEMS \\ Warton, UK
}

Various activities will be used to assess, understand and improve system reliability during development. The outcomes from each activity are not mutually exclusive as the same design weakness can be revealed through different activities. The cost associated with each activity, and the outcome, varies. This paper considers trade-offs between different types of costs and benefits and develops an approach to scheduling reliability activities.

\section{Introduction}

The motivation for this research is the need to manage reliability development programmes for aerospace systems. These may last from a few years to as many as ten years, during which time dozens of reliability activities are conducted, with the cost of an activity ranging from a few thousand pounds to a million pounds a month. Throughout, managers must consider trade-offs between the expected cost and benefits associated with each activity. Delaying activities has the possible benefit that it may become unnecessary to perform it, however, fundamental re-designs of a system late in programme can substantially increase costs. Explicitly modelling these costs are essential. Research into the development of decision support systems to help determine a cost effective reliability development programme is very much neglected. Models addressing optimal termination of testing within a cost-benefit framework exist [1], but stop short of determining an optimal portfolio of activities. This paper extends the model reported in [2].

This paper presents a statement of the decision problem in section 2, a general approach to obtaining optimal sets of activities in section 3 and provides an illustrative example in section 4 , concluding with suggestions for further work in section 5.

\section{Decision Problem}

We assume $\mathbf{J}$ distinct reliability activities could be performed on the system design to enhance reliability, where activity $i$ is denoted by $A_{i}$ and the decision to conduct no activities is denoted by $A_{0}$. The costs and benefits realised after a sequence of activities, such as activity $i$ and $j$ are denoted as $m\left(A_{i}, A_{j}\right)$. First, an activity is selected 
then a realisation is made, whereby the current measure of reliability increases (decreases) if the likelihood of potential faults due to a design weakness is reduced (increased), or remains unchanged if the activity was uninformative. Therefore, the outcome can take a range of possible values. The impact on the estimate of reliability is only one measure of interest; others include calendar time to complete each activity and the associated cost.

Typically, the objective is to minimise costs, such as in (1) while obtaining a minimum level of reliability. While project time can be factored into the costs associated with activities, it is desired to make an explicit account of the project duration. As such, we may seek to minimise calendar time with some constraint on costs (or vice versa) or some hybrid strategy.

$$
\operatorname{Min}_{\forall y_{i} \in[0, \mathrm{~J}]}\left\{\mathrm{m}\left(\mathrm{A}_{y_{1}}, \mathrm{~A}_{y_{2}}, \ldots, \mathrm{A}_{y_{n}}\right)\right\}
$$

\section{General Approach to Optimal Set of Activities}

\subsection{Stochastic Model of Reliability and Expert Judgement}

We assume the system has enumerable design weakness that will result in the failure of items in operation if not corrected. The purpose of the development activities is to detect these weaknesses or confirm they do not exist. Moreover, we assume the failure time characteristics of design weakness are different, but not unique, and so we can classify types of design weakness based on failure time distributions. We assume $\mathrm{C}$ classes. Hence, we model the reliability function of the time to first failure of the system as:

$$
R\left(t \mid N_{k}\right)=\left[R_{k}(t)\right]^{N_{k}}
$$

where $\mathrm{N}_{\mathrm{k}}$ is the number of design weakness in class $\mathrm{k}$ and $\mathrm{R}_{\mathrm{k}}(\mathrm{t})$ is the probability a design weakness in class $\mathrm{k}$ is realised after time $\mathrm{t}$.

Inference concerning $\mathrm{R}_{\mathrm{k}}(\mathrm{t})$ is supported through either expert judgement or historical data, while inference on $\mathrm{N}_{\mathrm{k}}$ is supported through elicitation of expert judgement to construct prior distributions. The elicitation involves relevant engineers assessing possible faults that may exist within the design, which they refer to as concerns, and assigning a probability for each to measure the likelihood that each concern will be realised as a failure in operation. We denote the probability that the $i^{\text {th }}$ concerning in class $k$, of which there are $I_{k}$, by $\lambda_{i k}$ then, by averaging over (2) with the subjective probabilities, we obtain the following reliability function for the time to first failure of the system:

$$
\mathrm{R}(\mathrm{t})=\prod_{\mathrm{k}=1}^{\mathrm{C}} \prod_{\mathrm{i}=1}^{\mathrm{I}_{\mathrm{K}}}\left[\mathrm{R}_{\mathrm{k}}(\mathrm{t}) \lambda_{\mathrm{ik}}+\left(1-\lambda_{\mathrm{ik}}\right)\right]
$$


Given this reliability function, we require a target in-service reliability requirement. For example, the realisation of the first failure of the system occurring after 1000 operating hours being above a specified quantity, $\mathrm{R}_{0}$. The effectiveness of each activity is assessed a priori during elicitation, whereby each activity is assessed against each concern for the probability of detecting the fault if it exists. The probability of activity $\mathrm{j}$ mitigating concern $\mathrm{i}$ from class $\mathrm{k}$ is denoted by $\mathrm{p}_{\mathrm{ijk}}$. This is used to assess the optimal set of activities.

\subsection{Selecting Set of Activities}

Assume $\mathbf{J}$ possible reliability activities from which to select a subset and create a development programme. Each activity (e.g. $\mathrm{j}^{\text {th }}$ ) has an associated duration of project time (denoted by $x_{\mathrm{j}}$ ) required to complete and we assume all activities will be conducted in series.

If a combination of activities can be found so that the estimated reliability exceeds the required reliability $\left(\mathrm{R}_{0}\right)$ within the time constraint (denoted by $\mathrm{T}_{0}$ ) then we call this combination a solution. When there is more than one solution, we select the solution that also gives minimal cost (i.e. expenditure for all the activities). If two solutions also have the same minimal cost, then we select the one that takes the least time. If two solutions also have the same least time, we select the one that gives the best reliability (i.e. is expected to mitigate the greatest number of concerns). We aim to minimise costs while achieve our reliability in (4) and completing the project in time as in (5). For simplicity we have remove the non-systematic failure effect.

$$
\begin{aligned}
& \prod_{\mathrm{k}=1}^{\mathrm{C}} \prod_{\mathrm{i}=1}^{\mathrm{I}_{\mathrm{K}}}\left[\mathrm{R}_{\mathrm{k}}(\mathrm{t}) \lambda_{\mathrm{ik}} \prod_{\mathrm{j}=1}^{\mathrm{J}}\left(1-\mathrm{p}_{\mathrm{jik}}\right)^{\Theta_{\mathrm{j}}}+\left(1-\lambda_{\mathrm{ik}} \prod_{\mathrm{j}=1}^{\mathrm{J}}\left(1-\mathrm{p}_{\mathrm{jik}}\right)^{\Theta_{\mathrm{j}}}\right)\right] \geq \mathrm{R}_{0} \\
& \sum_{\mathrm{j}=1}^{\mathrm{J}} x_{\mathrm{j}} \Theta_{\mathrm{j}} \leq \mathrm{T}_{0}
\end{aligned}
$$

where $\Theta_{j}$ is an indicator variable that is 1 if activity 1 is conducted and 0 if not. Therefore, (4) is the probability that the time to first failure of a system will be after time $t$ given a portfolio of reliability activities have been completed. This is provides a constraint as we have a minimal reliability target, i.e. $\mathrm{R}_{0}$, to achieve. Programme costs are minimised subject to the reliability constraint and project time expressed in (5). We could formulate the problem in terms of minimising project time, if that were desired.

\subsection{Sequencing the Activities}

We have a stopping rule that states if our estimated reliability is above a target value then terminate development. The effectiveness of each activity is subject to change throughout development. While each activity is distinct in its approach to detecting a design weakness, more than one activity could detect the same weakness, as such, if an activity detects a weakness, provoking a corrective action and removing it, then the weakness is precluded from being detected from all remaining activities. 
To solve this problem, we propose initially sequencing the activities by the ratio of cost to benefit, so that the most cost effective activity is sequenced first. Denote the $i^{\text {th }}$ activity scheduled as $S_{i}$. Therefore, denoting the cost of activity $j$ as $g_{j}$, select the $\mathrm{j}$ such that the cost-benefit ratio is:

$$
\max _{j}\left\{\frac{\prod_{k=1}^{\mathrm{C}} \prod_{\mathrm{i}=1}^{\mathrm{I}_{\mathrm{K}}}\left[\mathrm{R}_{\mathrm{k}}(\mathrm{t}) \lambda_{\mathrm{ik}}\left(1-\mathrm{p}_{\mathrm{jik}}\right)+\left(1-\lambda_{\mathrm{ik}}\left(1-\mathrm{p}_{\mathrm{jik}}\right)\right)\right]-\prod_{\mathrm{k}=1}^{\mathrm{C}} \prod_{\mathrm{i}=1}^{\mathrm{I}_{\mathrm{K}}}\left[\mathrm{R}_{\mathrm{k}}(\mathrm{t}) \lambda_{\mathrm{ik}}+\left(1-\lambda_{\mathrm{ik}}\right)\right]}{\mathrm{g}_{\mathrm{j}}}\right\}
$$

Which is approximately equal to the following.

$$
\max _{\mathrm{j}}\left\{\sum_{\mathrm{k}=1}^{\mathrm{C}} \sum_{\mathrm{i}=1}^{\mathrm{I}_{\mathrm{k}}} \lambda_{\mathrm{ik}}\left[\mathrm{R}_{\mathrm{k}}(\mathrm{t}) \mathrm{p}_{\mathrm{jik}}-\left(1-\mathrm{p}_{\mathrm{jik}}\right)\right]-\ln \left(\mathrm{g}_{\mathrm{j}}\right)\right\}
$$

If there is little difference between the failure time distributions across categories then the objective function becomes:

$$
\max _{j}\left\{R(t) E[D(j)]-E[D(0)-D(j)]-\ln \left(g_{j}\right)\right\}
$$

where $D(j)$ is the number of concerns mitigated by activity $j$ and $D(0)$ is the number of faults in the design prior to the start of development.

Once the first activity has been realised then the likelihood's associated with each concern are subject to change. We denote the updated probabilities by $\lambda_{\mathrm{ik}}\left(\mathrm{S}_{1}\right)$ and we have a new problem, with perhaps a higher or lower expected number of concerns being realised if the design were released, and a set of J-1 activities. We repeat the above process to sequence the next activity. Of course, following the realisations from each activity we may wish to consider introducing new activities to those identified.

\section{Illustrative Example}

The example is a typical application of this approach. Fifteen concerns had been identified during an elicitation. There was only one class of fault. Inferences from historical data supported an exponential distribution for the realisation of any fault with a hazard rate of 20 failures/1000 operating hours. The reliability function of the time to first failure of the system prior to conducting any activities is:

$$
\begin{aligned}
\mathrm{R}(\mathrm{t}) & =\mathrm{E}_{\mathrm{N}}\left[\mathrm{e}^{-0.02 \mathrm{tN}}\right] \\
& =\prod_{\mathrm{i}=1}^{15}\left(1-\lambda_{\mathrm{i}}+\lambda_{\mathrm{i}} \mathrm{e}^{-0.02 \mathrm{t}}\right)
\end{aligned}
$$


Each engineering expert assessed the likelihood of each activity mitigating each concern provided from a list of 22 reliability activities. A cost was associated with each reliability activity as well as an expected duration. The reliability requirement was in the form of an estimate of the chance of the system operating without failure by 1000 operating hours. The general form of the reliability function of the time to first failure of the system following a portfolio of activities is:

$$
\mathrm{R}(\mathrm{t})=\prod_{\mathrm{i}=1}^{15}\left(1-\left(1-\mathrm{e}^{-0.02 \mathrm{t}}\right) \lambda_{\mathrm{i}} \prod_{\mathrm{j}=\mathrm{i}}^{22}\left(1-\mathrm{p}_{\mathrm{ij}}\right)^{\Theta_{\mathrm{j}}}\right)
$$

The minimum cost is 56 cost units, has a total activity time of about 145 development time units to conduct the four identified activities $(1,2,12,17)$ and meets a target reliability of $85 \%$.

Figure 1 shows a contour plot, highlighting regions of joint costs and project time that will achieve specified reliability values. This plot was constructed for a target reliability of 0.85 and as such the contour reliability lines are all close to but exceed 0.85 . From Figure 1 the maximum cost (89) is approximately 50\% greater than the minimum cost (58). The minimum project time was 88 and the maximum 155 . As reliability increases, the impact on minimum costs is marginal. However, minimum project time is very sensitive to the target reliability. Moreover, the contour line at the minimum cost is very flat and implies project time could be reduced with little addition to overall cost.

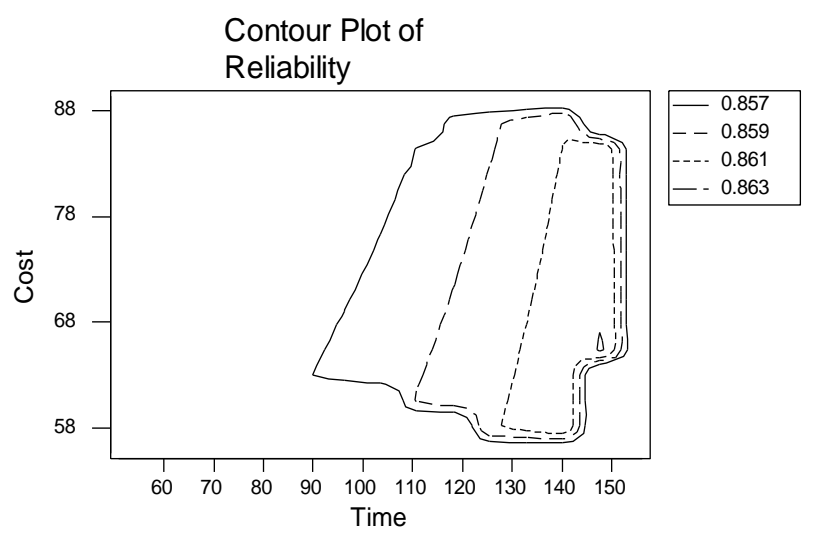

Figure 1 Contour line of reliability as a function of project time and cost

\section{Conclusions}

We have considered the relationship between the expected reliability from a development programme, the costs associated with the programme and the project time required to complete the schedule. The example illustrates the sensitivity of project time to reliability target, which highlights that blindly choosing the set of 
activities that minimise the expected project costs can result in longer than necessary projects. We are extending the model to incorporate a more flexible decision-making framework, formally accounting for the dependencies between the outcomes form activity.

\section{Acknowledgements}

This work has been conducted under the auspices of the REMM project and sponsorship of the UK DTI CARAD programme. The authors would like to thank all REMM industrial partners for their contributions which have informed the modelling framework.

\section{References}

1. Gaver D, Jacobs P, Seglie E Balancing "Test-Fix-Test" and Released System Reliability Under a Fixed Budget, Proc. $3^{\text {rd }}$ Int..Conf. on Mathematical Methods in Reliability, 2002; pp 251-254

2. Johnston, W, Quigley J and Walls L, Optimal Allocation of Reliability Activities to Mitigate Faults during New Product Development, submitted to IMA Modelling Industrial Maintenance and Reliability 